\title{
Sistemas de producción agroforestales. Capacitación y análisis en:
}

"conceptos generales y definiciones"

\section{Agroforestry production systems. Training and analysis "general concepts and definitions"}

Iglesias $\mathrm{JM}^{1}$

1Estación Experimental de Pastos y Forrajes "Indio Hatuey" Matanzas, Cuba iglesias@indio.atenas.inf.cu

CONFERENCIA DEL I SEMINARIO INTERNACIONAL DE GANADERIA AGROECOLÓGICA REALIZADO EN VILLAVICENCIO COLOMBIA, 11 y 12 de noviembre de 2010

\section{RESUMEN}

Las graves afectaciones que han tenido, de forma general, los recursos naturales y la actual crisis económica y social que atraviesan diversos países, han revitalizado el interés por lograr un desarrollo acelerado y sostenido de la agricultura, el cual solo se conseguirá en la medida en que las estrategias de producción sean congruentes con el uso racional del ecosistema. En este contexto, el visualizar la actividad agropecuaria en sistemas agroforestales constituye un enfoque válido, necesario y actual en la investigación y la capacitación para el desarrollo pecuario de los trópicos. Los sistemas de producción agroforestales se definen como una serie de sistemas y tecnologías del uso de la tierra en las que se combinan árboles con cultivos agrícolas y/o pastos, en función del tiempo y el espacio para incrementar y optimizar la producción en forma sostenida. Estos sistemas pueden contribuir a solucionar problemas en el uso de los recursos naturales debido a las funciones biológicas y socioeconómicas que cumplen. Desde el punto de vista biológico, la presencia de los árboles favorece los sistemas de producción en aspectos tales como el mantenimiento del ciclaje de nutrimentos y el aumento en la diversidad de especies. Además, debido a la estructura vertical proporcionada por los árboles y otras especies leñosas, pueden convivir plantas y cultivos con diferentes requerimientos de luz; asimismo, los árboles protegen al suelo de los efectos del sol, el viento y las fuertes lluvias que caracterizan al trópico. El uso de prácticas agroforestales también puede proveer beneficios económicos y socioculturales, 
entre los cuales se pueden mencionar la disminución de los riesgos económicos por la diversificación de la producción y la creación de nuevos puestos de trabajo en tareas de viveros, siembras, mantenimiento de áreas, etc. Los primeros intentos para definir la agroforestería se remontan a 1977-1979, Combe y Budowski, la definían como "un conjunto de técnicas de manejo de tierras, que implican la combinación de los árboles forestales, ya sea con la ganadería, o con los cultivos". Sin embargo, la palabra agroforestería llegó a nuestro idioma proveniente del inglés agroforestry, por lo que en los últimos años se han expresado diversos criterios acerca de la verdadera definición de este concepto. Se ha venido usando indistintamente las palabras: agrosilvopastoril, sistemas agrosilvopastoriles, agrodasonomía y agrosilvicultura, entre otras. En el desarrollo de este artículo reseña se hará uso de los términos agroforestería y sistemas agroforestales, por ser los más usados en la literatura revisada para definir las técnicas agroforestales. También se hace necesario aclarar que se abordarán los sistemas agroforestales más relacionados con los cultivos agrícolas y sus combinaciones, debido a que en años anteriores se publicó un artículo en esta misma revista acerca de los sistemas silvopastoriles para la ganadería.

Palabras clave: Revisión, funciones, agroforestería, aprendizaje.

\section{ABSTRACT}

The serious effects that have, in general, natural resources and economic and social crisis that cross several countries, have revitalized the interest in an accelerated and sustained development of agriculture, which can only be achieved to the extent production strategies that are consistent with the rational use of the ecosystem. In this context, see the agricultural activities in agroforestry systems is a valid approach, necessary and current research and training in livestock development in the tropics. Agroforestry production systems are defined as a series of systems and technologies in land use that combines trees with crops and/or pasture, depending on the time and space to increase and optimize production steadily. These systems can help solve problems in the use of natural resources due to biological and socio-economic functions they perform. From the 
biological point of view, the presence of trees encourages production systems in areas such as maintaining nutrient cycling and increased species diversity. In addition, because the vertical structure provided by trees and other woody plants and crops can coexist with different light requirements, also, the trees protect the soil from sun, wind and heavy rains that characterize the tropics. The use of agroforestry can also provide economic and sociocultural benefits, among which we can mention the reduction of economic risks by diversifying production and creating new jobs in nursery work, planting, maintenance areas, etc. Early attempts to define agroforestry back to 1977-1979, Combe and Budowski described it as "a set of land management techniques, which involve the combination of forest trees, either livestock or crops." However, the word came into our language Agroforestry English from agroforestry, so in recent years have expressed various views about the true definition of the concept. He has been using the words interchangeably agroforestry, agroforestry systems, agrodasonomy and agroforestry, among others. In developing this article will review the terms of use agroforestry and agroforestry systems, because they are widely used in the literature review to define agroforestry techniques. It is also necessary to clarify that agroforestry systems will be addressed more closely related to agricultural crops and their combinations, because in previous years, published an article in this magazine about silvopastoral systems for cattle.

Keywords: Review, functions, agroforestry, learanig.

\section{DEFINICIÓN DE AGROFORESTERÍA}

A través de los años se han dado numerosas definiciones de agroforestería o sistemas agroforestales. El International Council for Research in Agroforestry (1983) la define como: "Sistema sostenido del manejo de la tierra que aumenta su rendimiento total, combina la producción de cultivos con especies forestales y/o animales, en forma simultánea o secuencial sobre la misma superficie de terreno, y aplica prácticas de manejo que son compatibles con las prácticas culturales de la población local". 
Maydell, (1984) propone referirse a agroforestería "sólo en aquellos casos en que los árboles y arbustos, así como los cultivos agrícolas y los animales de pastoreo, se encuentran juntos sobre una determinada parcela de terreno, en forma tal que se pueda demostrar una influencia ecológica mutua”. Budowski, (1984) señala que "el objetivo principal de estos sistemas es optimizar la producción total por unidad de superficie, respetando el principio de rendimiento sostenido." Por su parte, Somarriba, (1998) define la agroforestería como "una forma de cultivo múltiple en la que se cumplen tres condiciones fundamentales: 1) existen al menos dos tipos de plantas que interactúan biológicamente; 2) al menos uno de los componentes es una leñosa perenne y 3) al menos uno de los componentes es una planta manejada con fines agrícolas (incluyendo pastos)". En la revista Agroforestry Systems se define (Anon, 1981) el siguiente concepto de agroforestería: "Sistema de uso de la tierra donde leñosos perennes interactúan bioeconómicamente en una misma área con cultivos y animales. Estos elementos pueden estar asociados en forma simultánea o secuencial, en zonas o mezclados. Las formas de producción son aplicables tanto en ecosistemas frágiles como estables, a escala de campo agrícola, finca o región, a nivel de subsistencia o comercial. El objetivo es diversificar la agricultura migratoria, aumentar el nivel de materia orgánica en el suelo, fijar nitrógeno atmosférico, reciclar nutrimentos, modificar el microclima y optimizar la producción del sistema, respetando el principio de rendimiento sostenido. Se exige compatibilidad con las condiciones socioculturales de la población y debe servir para mejorar las condiciones de la región".

Montagnini et al., (1992) definen un sistema agroforestal como un sistema agropecuario cuyos componentes son los árboles, los cultivos o los animales, y que presenta los atributos de cualquier sistema: límites, componentes, ingresos y egresos, interacciones, una relación jerárquica con la organización de la finca y una dinámica. El límite define los bordes físicos del conjunto; los componentes son los elementos físicos, biológicos y socioeconómicos; los ingresos y egresos son la energía o la materia que se intercambia entre diferentes sistemas; las interacciones son la energía o la materia que se intercambia entre los componentes del sistema y la jerarquía indica la posición de éste con respecto a 
otros sistemas y las relaciones entre ellos. Kass, (1992) conceptuando el término en cuestión y haciendo énfasis en algunas de sus características y ventajas, considera que la agroforestería: 1. Es un nombre colectivo para sistemas que involucran el uso de árboles con cultivos y/o animales en la misma unidad de terreno. 2. Pone énfasis en el empleo de árboles y arbustos indígenas y de uso múltiple. 3. Combina la producción de egresos múltiples con la protección de la base de recursos. 4. Es muy apta para condiciones de bajos insumos y ambientes frágiles. 5. Es estructuralmente más compleja que el monocultivo. 6. Involucra la interacción de valores socioculturales en mayor grado que la mayoría de los demás sistemas del uso de la tierra.

Si se hace un análisis de los diferentes conceptos de agroforestería citados anteriormente, se destaca como condición indispensable la presencia del árbol o arbusto (leñosa perenne) para que el sistema agrícola sea forestal; sin embargo, esta leñosa debe aparecer indistintamente asociada biológicamente a otros cultivos (perennes o anuales) y en ocasiones (no necesariamente) a animales. En las condiciones actuales de desarrollo de los países tropicales, como es el caso de Cuba, se impone hacer una valoración objetiva acerca de la utilización de estos sistemas, ya que los componentes arbóreos cumplen un sinnúmero de objetivos, tales como: producción de madera, mejoramiento del microclima y el suelo, aporte de materia orgánica, fijación de nitrógeno y captación de dióxido de carbono, producción de follaje y otros productos alimenticios y medicinales, gomas, resinas y fibras. Con la aplicación de las técnicas agroforestales las actividades agrícolas y ganaderas adquieren un carácter permanente; su desarrollo no requiere de altos insumos, ya que el uso de la maquinaria es mínimo, ni tampoco se precisa de altas dosis de fertilizantes ni pesticidas para mantener el sistema de producción de forma sostenible. En este sentido, los sistemas agroforestales se presentan como un reto para la esfera agropecuaria, por ser una solución económicamente viable, socialmente aceptada y que no produce daños ambientales; por otra parte, generan tecnologías poco costosas, lo cual es un esfuerzo importante si se tiene en cuenta la señal de alarma a nivel mundial, debido al fracaso de la Cumbre de la Tierra realizada en Nueva York en el año 1997. 


\section{FUNCIONES QUE DESEMPEÑAN LOS ÁRBOLES EN LOS SISTEMAS AGROFORESTALES}

Un método de uso de la tierra es sostenible si éste no produce deterioro de la capacidad productiva del sitio. La presencia de árboles provee a los sistemas agroforestales algunas características que pueden favorecer la productividad y la perdurabilidad de los mismos. Camacho y Yaelis, (1992) definen la intervención del árbol en los sistemas agroforestales por diferentes niveles:

Nivel de producción: el árbol puede poner a la disposición del agricultor una gran cantidad de productos destinados tanto al mercado como al consumo familiar, entre los que se señalan los alimentos para el hombre (frutos, nueces, hojas), los materiales de construcción (madera, cujes, etc.), el combustible (leña y carbón), el alimento para animales (forraje, frutos) y otros productos como son los medicinales, las bebidas estimulantes, los aceites, los perfumes, los colorantes, las gomas, el papel, las fibras textiles $u$ otras, los insecticidas y pesticidas, materiales de artesanía, postes para cercados y otros objetos de uso doméstico.

Nivel de servicios: además de los productos directamente aprovechables, el árbol aporta una serie de servicios a la agricultura que son difícilmente estimables en términos de dinero, sin embargo, son esenciales e incluso más numerosos que los directos. Entre los posibles efectos beneficiosos de los árboles sobre los suelos (Nair, 1989) se destacan: la adición de materia orgánica, el aumento del contenido de nitrógeno, su influencia sobre cantidad y distribución de lluvias y su aporte de nutrimentos, reducción de la pérdida de suelo y de nutrimentos, la absorción de nutrimentos en las capas profundas y su deposición en la superficie, la conservación de nutrimentos que podrían perderse por lavado de los suelos, la liberación (por medio del manejo) de nutrimentos en el momento requerido por los cultivos, la mejora de las propiedades físicas (retención de agua, drenaje, etc.), el aumento de la biomasa de las raíces, sustancias promotoras del crecimiento, asociaciones microbianas, la mejora de la calidad de la hojarasca, a través de la diversidad de especies, la creación de un microclima favorable y la moderación de 
efectos en condiciones de acidez, alcalinidad u otras condiciones desfavorables de los suelos. En relación con las plagas de los cultivos, los árboles aumentan la diversidad del entorno y se convierten en un instrumento de equilibrio y control biológico de las plagas. Se conocen como otros servicios la conservación de riberas y taludes, las cercas vivas, los tutores vivos y la utilización complementaria de recursos.

Nivel sociocultural: el árbol desempeña un importante rol en la vida social y cultural de los pueblos, ya que tiene gran influencia en los derechos de tenencia de la tierra, así como significado simbólico y hasta mítico en algunas regiones. Utilizado como ornamental mejora el hogar y el cuadro de vida del hombre en el campo, y además, prácticamente no hay una especie de árbol que no tenga usos medicinales. Ciertos árboles combinan las funciones de los tres niveles. Por lo que se denominan de uso múltiple (Geilfus, 1989).

\section{CONDICIONES NECESARIAS PARA LA SIEMBRA DE ÁRBOLES EN LOS SISTEMAS AGRÍCOLAS}

En ocasiones, incluso donde los árboles son muy escasos, los agricultores pueden estar no dispuestos a cultivarlos. Tradicionalmente han fracasado muchos intentos de reemplazar los complejos sistemas tradicionales de uso de la tierra, debido aparentemente a los riesgos del clima, plagas, dificultades en el manejo de los suelos y condiciones sociales y culturales que dificultan la aceptación de los nuevos sistemas (Office of Techonology Assessment, 1984). Se han hecho muchos intentos, con éxito variable, como son proyectos de reforestación a gran escala, reservas forestales, leyes para el control de la tala de bosques; sin embargo, a veces no se valoran adecuadamente los beneficios que se producen, además de otras limitaciones que existen. Según la FAO, (1984) las principales condiciones que se deben cumplir antes de que los agricultores planten árboles en sus sistemas agrícolas son:

Económicas: Tienen que existir suficientes recursos disponibles de tierra, semillas, capital y mano de obra para hacer posible el cultivo de los árboles y 
cubrir los gastos de plantación, cuidado, cosecha y comercialización de ellos y sus productos. Habitualmente se presenta secases de plantas, falta de control de calidad en la producción de semillas y arbolitos, falta de información sobre las técnicas de propagación, falta de incentivos y créditos adecuados para la reforestación, todo esto ligado a la falta de adecuada divulgación técnica. Los beneficios del cultivo y manejo de los árboles, deben superar los beneficios netos de estrategia, alternativas de ordenación de los recursos y la agricultura, así como los costos de producción.

Socioculturales: Los cambios que pueden traer consigo el cultivo de los árboles en cuanto a las relaciones de productividad y los modelos de propiedad de los recursos, se deben enmarcar dentro de las estrategias culturalmente aceptadas para la distribución de recursos. El valor social de los árboles debe coincidir con los valores que pueden imponer las gestiones e intervenciones de manejo adoptadas. Entre los beneficios socioeconómicos y culturales que provee el uso de prácticas agroforestales puede mencionarse la disminución de los riesgos económicos para el agricultor al lograr diversificar, la producción; el empleo de mano de obra familiar con una mejor integración de los miembros de la familia al proceso productivo y el mantenimiento de costumbres o prácticas de uso de la tierra, de fuerte arraigo cultural, en algunos casos.

Ambientales: Las intervenciones o gestiones de adaptación deben tener en cuenta el conocimiento adecuado de las condiciones de los sitios elegidos, la disponibilidad de agua, el régimen de temperatura, así como otras características del medio ambiente natural.

\section{CLASIFICACIÓN DE LOS SISTEMAS AGROFORESTALES}

La clasificación de los sistemas agroforestales es necesaria con el fin de proveer un marco conceptual que permita evaluarlos y desarrollar planes de acción para su mejoramiento (Nair, 1989) Los sistemas agroforestales han sido clasificados de diferentes maneras: según su estructura en el espacio, su diseño a través del tiempo, la importancia relativa y la función de los diferentes componentes, los 
objetivos de la producción y las características sociales y económicas prevalecientes. Combe y Budowski, (1979) se basan en los productos que se pueden obtener y el tipo de combinación entre los componentes. Otras clasificaciones se sustentan en la escala y en los objetivos de la producción: sistemas comerciales, de subsistencia e intermedios (Office of Technology Assessment, 1984). Martínez, (1989) identificó cuatro grupos de combinaciones o sistemas agroforestales posibles en los sistemas de fincas de pequeños $y$ medianos agricultores. Por su parte Nair (citado por Kass, 1992 y Montagnini et al., 1992) recomienda una clasificación en la que se tomen en cuenta los aspectos estructurales y funcionales como base para agrupar los sistemas en cuatro grandes grupos (Tabla 1).

Como se aprecia, estos grupos se subdividen de acuerdo con criterios de arreglo espacial (sistemas mixtos, densos, en franjas, etc.), de arreglo temporal (secuenciales, coincidentes, interpolados, etc.), funciones de los componentes (leña, forraje, cercos vivos, conservación de suelos, etc.), zonas agroecológicas donde se desarrollan (zonas altas, semiáridas, etc.) y aspectos socioeconómicos (altos o bajos insumos, otros.).

Tabla 1. Combinaciones agroforestales posibles en los sistemas de finca de pequeños y medianos agricultores (Martínez, 1989).

Grupo 1. Árboles con cultivo

1. Disperso

2. Intercalado

a. Sombra inicial

b. Sombra permanente

c. Cultivos secuenciales

3. En callejones

4. Líneas alternadas

5. Árboles Taungya
Grupo 2. Árboles para protección

1. Cercas vivas o árboles en cerco

2. Cortinas rompevientos

3. Árboles en contorno

4. Barreras vivas

5. Estabilización/recuperación de suelos

6. Protección de cauces y nacimientos

Grupo 3. Árboles en rodales compactos Grupo 4. Árboles en potreros

1. Bosque de producción de madera.

1. Árboles dispersos

2. Árboles en grupo

2. Bosque energético

3. Árboles en grupo

3. Banco de forrajes

4. Huertos caseros 
Budowski, (1990) los clasifica como: a) Arboles de sombra sobre café y cacao; a veces incluyen un estrato más alto de árboles maderables. b) Arboles asociados con cultivos anuales o semiperennes. Incluye el sistema secuencial conocido como Taungya. c) Arboles en potreros bajo diversas modalidades y cumpliendo varias funciones. d) Cercas vivas, establecidas principalmente por estacas grandes. e) Cortinas rompevientos. f) Huertos caseros tropicales mixtos. g) Mejoramiento del rastrojo en la fase de recuperación, en terrenos donde se practica la agricultura nómada. h) Modalidades secuenciales donde se alternan pastos con bosques secundarios manejados para la producción de madera. i) Cultivo en franjas o en callejones. Por otra parte, Montagnini et al., (1992) presentaron una clasificación basada en el tipo de componente incluido y la asociación entre los componentes. De ese modo, los sistemas agroforestales descritos son:

Sistemas agroforestales secuenciales, donde se da una relación cronológica entre las cosechas anuales y los productos arbóreos, o sea, que los cultivos y las plantaciones de árboles se suceden en el tiempo. Estos incluyen: a) Formas de agricultura migratoria con intervención o manejo de barbechos y .b) Sistemas Taungya.

Sistemas agroforestales simultáneos, los que consisten en la integración simultánea y continua de cultivos anuales o perennes, árboles maderables, frutales o de uso múltiple y/o ganadería. Estos abarcan: a) Asociaciones de árboles con cultivos anuales o perennes. b) Huertos caseros mixtos. Y c) Sistemas agrosilvopastoriles.

Sistemas agroforestales de cercas vivas y cortinas rompevientos. Estos consisten en hileras de árboles que determinan una propiedad o sirven de protección para otros componentes o sistemas. De acuerdo con el tipo de cultivo asociado, la función principal del componente forestal y su distribución en el espacio y el tiempo, Fassbender, (1993) llega a un arreglo múltiple y complicado de los sistemas agroforestales, los cuales incluyen: 
A. Sistemas silvoagrícolas 1. Sistemas Taungya o agrosilvicultura 2. Arboles de valor en los cultivos, 3. Árboles frutales en los cultivos y 4. Arboles productores de sombra en los cultivos y/o mejoradores de la fertilidad del suelo 5. Cercas vivas, 6. Cortinas rompevientos, 7. Cultivos en fajas o callejones, 8. Sistemas agroforestales múltiples y 9 . Huertos caseros.

B. Sistemas agrosilvopastoriles: 1. Cultivos y ganadería simultánea en plantaciones, 2. Arboles asociados a los cultivos y ganadería, y 3. Cercas vivas alrededor de comunidades rurales.

C. Sistemas silvopastoriles: 1. Pastoreo (o producción de forraje) en las plantaciones forestales, 2. Pastoreo (o producción de forraje) en bosques secundarios, 3. Arboles maderables en los pastizales, 4. Arboles de servicios en los pastizales, 5. Árboles frutales en los pastizales, 6. Arboles forrajeros, 7. Cercas vivas y 8 . Cortinas rompevientos.

Como se observa, las clasificaciones son disímiles, al igual que los criterios que se toman en cuenta para sus definiciones. Cada una de ellas tiene su mérito y su lógica; sin embargo, no todas son perfectas, por lo que es inadmisible tomarlas como patrones únicos de trabajo y reconocerlas universalmente para ser aplicadas en cualquier situación o entorno productivo. La clasificación de un sistema u otro siempre dependerá del propósito para el cual es destinado. En la presente reseña se analizará un grupo de sistemas agroforestales, que por su importancia y generalización a nivel mundial aparecen en casi todas las clasificaciones anteriormente reseñadas: los sistemas Taungya, los huertos caseros mixtos o tradicionales, los cultivos en callejones, los árboles de sombra sobre cultivos perennes y la agricultura migratoria con manejo de barbechos.

\section{SISTEMAS TAUNGYA}

Este sistema se basa en plantaciones forestales asociadas con rotaciones de cultivos temporales, cuyo objetivo es la producción de madera en su etapa final. El cultivo agrícola se limita a un corto período (1-4 años) hasta que los árboles plantados cierran su dosel (Figura 1). 


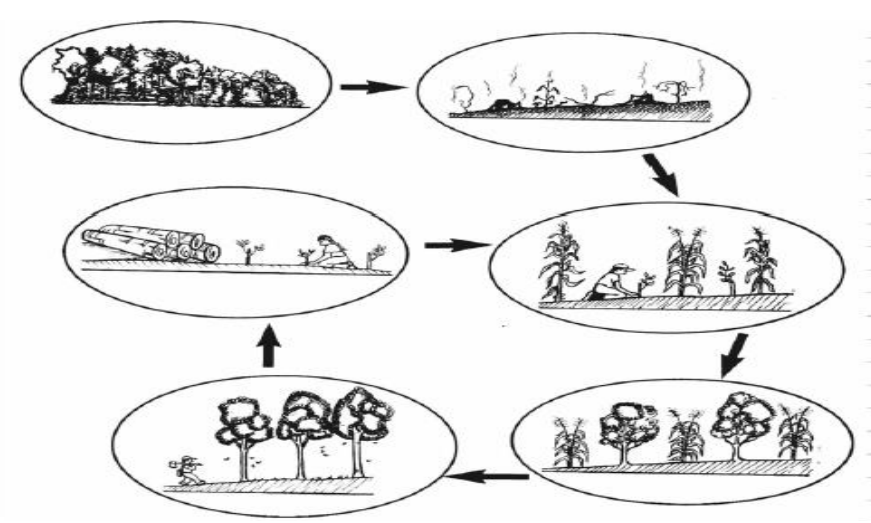

Figura 1. Esquema secuencial de un sistema Taungya

Tiene su origen en Birmania (Lamprecht, 1990) a partir de las reformas implantadas por el botánico alemán, Dr. Dietrich Brandis, al sistema de tumba y quema que existía en las plantaciones de teca (Tectona grandis) de ese país. De esta forma, el sistema de agricultura migratoria que aplicaba la tala y la quema se convirtió en una herramienta de transformación de los bosques en plantaciones forestales. El sistema Taungya se llevó de Birmania a otras colonias inglesas y europeas en Asia, de donde pasó a África. A pesar de los estudios realizados en América Central desde los años 60 de este siglo (Aguirre, 1963; Lucas, et al., 1995; Platen, 1996), el sistema todavía no ha sido adoptado notablemente en la región.

Con respecto al sistema Taungya, aún existen divergencias desde el punto de vista de su clasificación. En este sentido Combe y Budowski, (1979) aportaron los siguientes elementos: 1. El producto principal del sistema es la madera, 2. La combinación de agricultura y forestería es temporal y 3. La distribución espacial es regular (generalmente en hileras). Este sistema permite una mayor utilización del espacio, a la vez que reduce el costo y la limpieza inicial de las plantaciones al compararlo con las plantaciones forestales establecidas sin agricultura. Tradicionalmente se cultivan granos básicos, aunque en los últimos años se están incluyendo raíces, tubérculos y hortalizas (Schlönvoigt, 1998).

Beer et al., (1994) distinguen entre Taungya estatal y Taungya privado. En el primero el estado controla el manejo del sistema en terrenos extensos, con la 
participación de la población rural; mientras que el privado se realiza en terrenos propios del productor, quien es el dueño, tanto de los árboles como de los cultivos.

En los sistemas Taungya del sureste de Asia, el Pacífico y África las especies de árboles más utilizados son: Cupressus lusitanica, Eucalyptus deglupta, Gmelina arborea, Pinus caribaea, Pinus kesiya, Pinus patula y T. grandis (Nair, 1984; Young, 1989). En América Latina, que incluye a México, Brasil, Guatemala, Costa Rica, Haití y Cuba (Combe y Budowski, 1979; Lojan, 1979; FAO, 1984; Orquín, et al., 1988) son usadas las especies Cordia alliodora, Cordia goeldiana, Gliricidia sepium, Guazuma ulmifolia, Leucaena leucocephala, Azadirachta indica, Cassia siamea, G. arborea, T. grandis, Cedrela odorata, Hibiscus elatus y Pinus cubensis, entre otras muchas.

El sistema Taungya como técnica agrosilvícola fue practicado en Cuba en forma aislada antes de la década del sesenta. Sin embargo, en los últimos 20 años se ha difundido su práctica, pues se evidenciaron las ventajas del cultivo intercalado en las plantaciones, lo que posibilita la producción de alimentos y simultáneamente garantiza la sobrevivencia de las plantaciones. Especial atención se ha venido prestando al cultivo de granos de ciclo corto y cucurbitáceas durante el establecimiento de los sistemas silvopastoriles en las provincias de La Habana y Holguín (Simón, 1998). Esta modalidad de "taungya cubano" consiste en la siembra de maíz, frijoles, calabaza, melón, pepino y otros cultivos, simultáneamente con $L$. leucocephala y otras arbóreas forrajeras en las vaquerías y unidades de desarrollo seleccionadas para la implementación del silvopastoreo a gran escala.

Durante la fase crítica del establecimiento de las arbóreas (primeros 80-100 días) los obreros de las unidades pecuarias se dedican a la limpieza de los cultivos sembrados, lo que a su vez beneficia el crecimiento de los árboles asociados a estos. Una vez realizada la cosecha de los cultivos de ciclo corto, el terreno queda listo para la siembra de los pastos del sistema, los cuales no deben interferir en el crecimiento de los árboles, ya que estos han alcanzado para esa etapa una altura adecuada y han desarrollado un sistema radical fuerte que les permite su posterior 
crecimiento y explotación. La diferencia entre esta modalidad de Taungya y el sistema birmano tradicional consiste en que el futuro de los árboles no es la producción de madera, sino el ramoneo de los animales, por lo que la siembra de cultivos se realiza sólo una vez y son sustituidos inmediatamente por pastizales.

La práctica de este sistema ha producido ganancias importantes para los productores ganaderos involucrados en el establecimiento de árboles para la producción de leche y carne. En la actualidad los costos para establecer una hectárea de silvopastoreo con leucaena varían entre 198 y 255 pesos. Con la siembra de cultivos de ciclo corto durante el establecimiento de la plantación se obtienen entre 250 y 447 pesos/ha por concepto de venta de las cosechas (Martín, G., comunicación personal), por lo que en la mayoría de los casos se paga la inversión inicial, aun sin estar en explotación el sistema pecuario.

\section{HUERTOS CASEROS MIXTOS O TRADICIONALES}

Los huertos caseros mixtos tropicales ocupan un lugar muy singular en los sistemas agroforestales. Ningún otro es tan diverso en cantidad de especies y variedades, complejo y variado en estructuras y posibles asociaciones, ni tan completo en sus funciones como el huerto casero (Lok, 1998). Se trata de un complejo de plantas perennes o semiperennes que se encuentran en los alrededores de las casas de los finqueros o agricultores, integrado a la producción agrícola (tubérculos, fibras, hortalizas, frutas, estimulantes), ganadera (animales menores, inclusive abejas) y forestal (madera, leña, postes) e incluye generalmente plantas medicinales y ornamentales.

Los huertos caseros son muy comunes en todos los países y regiones tropicales y subtropicales y su tamaño es generalmente pequeño, menor que una hectárea (Fassbender, 1993). Estos sistemas se utilizan para cubrir las necesidades básicas de familias o comunidades pequeñas y ocasionalmente se venden algunos excedentes de producción. A pesar de que no existe un consenso universal sobre la definición del huerto casero Nair, (1993) lo define como "una asociación interna de árboles y/o arbustos de uso múltiple con cultivos anuales y 
perennes y animales en las parcelas de hogares individuales. El sistema es manejado por "mano de obra familiar". El huerto casero está compuesto por varias estructuras que se encuentran en él a partir de la división de su espacio en diferentes áreas de manejo (Figura 2).

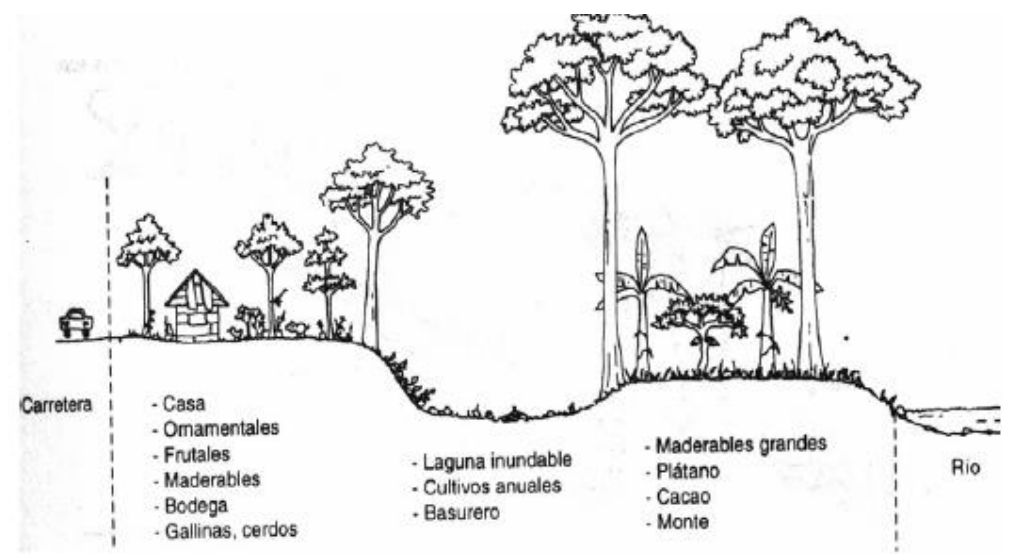

Figura 2. Caracterización gráfica del huerto casero mixto

Parecen ser universales en los huertos caseros las siguientes características de forma (estructura horizontal y vertical): 1. Un huerto bien desarrollado es una imitación del bosque tropical (Altieri, 1991). Este postulado se basa en que la semejanza del huerto casero con el bosque tropical está en el efecto de la forma del primero, la creación de nichos específicos, los diferentes niveles verticales y la competencia por la luz, así como en las funciones agroecológicas asociadas. 2. El huerto casero es una composición de diferentes áreas de manejo. Christant, (1990) caracteriza los huertos de acuerdo con su espacio libre y cultivado; en cada área hay una estructura horizontal y vertical propia, así como una combinación de especies y variedades de vegetación única. 3. La prevalencia de la sombra. Como consecuencia del número de especies arbóreas muchos de los huertos tropicales tradicionales están más sombreados que las fincas (Lok, 1998). Esto implica un microclima agradable y una preferencia de los campesinos por los cultivos resistentes a la sombra, que requieran poca inversión de mano de obra, cuando estos están destinados al autoconsumo.4. Los límites del huerto casero se definen por medio de una mezcla de factores geofísicos, biofísicos y sociales. A veces el huerto tiene límites geofísicamente claros cuando colinda con una carretera o camino, con el huerto vecino, etc.; otras veces el límite es biológico, como son los 
cambios de vegetación (termina el pasto y comienzan los cultivos en varios estratos), y por último el huerto casero se delimita por el uso de la mano de obra familiar y no muy intensiva; mientras que en la finca se contrata periódicamente. La principal característica de los huertos caseros mixtos en nuestro país lo constituye la amplia variedad de especies agrícolas, forestales y frutales, además de los animales que los conforman, lo que los convierte en sistemas agrosilvopastoriles integrados.

Entre las especies vegetales que más se utilizan en los huertos caseros mixtos se destacan: el ajo (Allium sativum), la piña (Ananas comosus), el ají (Capsicum sp.), la calabaza (Cucurbita pepo), la yuca (Manihot esculenta), el plátano (Musa sp.), el maíz (Zea mays), etc. Las especies frutales más preferidas por los campesinos son: el mango (Mangifera indica), la naranja agria (Citrus aurantium), el limón (Citrus limon), el aguacate (Persea americana), el coco (Cocos nucifera), la guanábana (Annona muricata) y la guayaba (Psidium guajava). Entre los árboles no frutales se destacan: el almácigo (Bursera simaruba), el matarratón ( $G$. sepium), la majagua ( $H$. elatus), la palma real (Roystonea regia) y el cedro ( $C$. odorata). No se descartan en este tipo de sistema agroforestal las plantas medicinales, muy necesarias en zonas alejadas de las áreas urbanas y centros asistenciales de salud. Entre las más prominentes se encuentran: la caña santa (Costus spiralis), la salvia americana (Lippia alba), el anís (Foeniculum vulgare), la manzanilla (Chrysantellum americanum), el toronjil de menta (Mentha citrasa), etc. La cría de animales puede ser diversa, en la que se destacan las gallinas criollas, los cerdos, los carneros y chivos y en ocasiones se encuentran bovinos de leche y equinos. En resumen, se puede afirmar que los huertos caseros mixtos tradicionales son una contribución a la serenidad y la felicidad del campesino, por su valor estético y recreativo, por ser una extensión de la casa, por constituir su taller de trabajo, el lugar donde se crían los hijos, una gran fuente de frutas, verduras y tubérculos, un medio de amortiguamiento en tiempos de escasez, una farmacia en vivo y además una fuente de recursos para la generación de ingresos a partir de los excedentes. 


\section{ÁRBOLES EN ASOCIACIÓN CON CULTIVOS PERENNES}

Los sistemas de explotación comercial con cocoteros, caucho o palma en asociación con cultivos y las plantaciones de árboles maderables con café y cacao pertenecen a esta categoría. En estas asociaciones se tiende a optimizar el uso de los recursos y aumentar la productividad por unidad de terreno; las condiciones de mercado son determinantes para el éxito. Generalmente, con los cultivos a pleno sol se logran cosechas más elevadas; sin embargo, las necesidades ecológicas son más altas, especialmente en fertilizantes (Fassbender, 1993). Estos sistemas representan una alternativa, ya que con la introducción de los árboles para sombra se puede llegar a suplir parte de las necesidades nutricionales del cultivo. Por otra parte, según Purseglove (citado por Beer, 1998) la sombra reduce la foto-síntesis, la transpiración, el metabolismo y el crecimiento; por consiguiente, decrece también la demanda de nutrimentos del suelo y así "se capacita" a un cultivo para que se mantenga en suelos de baja fertilidad. Los árboles de sombra para cultivos perennes deben reunir una serie de características (Jiménez y Vargas, 1998), entre las que resaltan: 1. Compatibilidad con el cultivo. 2. Sistema radical fuerte y resistente a los vientos. 3. Habilidad de propagación vegetativa por medio de estacas. 4. Habilidad para fijar nitrógeno. 5. Posesión de una copa rala. 6. Ramas y tallos no quebradizos y libres de espinas. 7. Tolerancia a la poda. 8. Alta producción de biomasa, con residuos vegetales de fácil descomposición. 9. Alta velocidad de rebrote. 10. Presencia de hojas pequeñas. 11. Producción de madera, frutos u otro producto de apreciable valor. 12. Resistencia a las plagas y las enfermedades.

En América Latina las especies más usadas en estos sistemas son las de los géneros Acacia, Albizia, Erythrina, Inga, Leucaena, Crotalaria, Sesbania, Cedrela, Cordia, Gliricidia, Calophyllum, Ficus, Swietenia, etc. En Cuba la asociación de árboles con cultivos perennes está entre las técnicas agroforestales tradicionales de mayor antigüedad y representatividad, ejemplo de ello lo constituyen las plantaciones cafetaleras de las principales zonas montañosas del Oriente, Centro y Occidente del país, las cuales se han fortalecido en los últimos años gracias al 
programa de recuperación cafetalera y el denominado "Plan Turquino", diseñado para impulsar el desarrollo económico y social de la montaña. En la actualidad hay alrededor de 130000 has dedicadas al café. Paralelamente a la producción cafetalera, la producción de cacao también se basa en el uso de los árboles como proveedores de sombra para el cultivo base. Se estima que el cacao se introdujo en Cuba en 1540 y en la actualidad también pasa por un proceso de recuperación, con la introducción de nuevos clones, medidas de conservación de los suelos, etc. Los árboles más usados como sombra de estos dos importantes cultivos son: $G$. sepium (matarratón), L. leucocephala (leucaena), Erythrina poeppigiana (búcare) y Albizia saman (algarrobo). En los últimos años, con la introducción y extensión de estos cultivos en ecosistemas no tradicionales, se ha generalizado el uso de otras especies como P. caribaea y P. cubensis (Renda et al., 1997).

\section{AGRICULTURA MIGRATORIA CON INTERVENCIÓN O MANEJO DE BARBECHOS MEJORADOS}

La agricultura migratoria es un sistema en el cual el bosque se corta y se quema y la tierra se cultiva durante pocos años; después del período de cultivo continúa una fase de barbecho. Esta fase es bastante más larga que la del cultivo (5-20 años de barbecho y 2-3 años de cultivo). En estos sistemas de uso transitorio de la tierra (Figura 3) se realiza una rotación de parcelas en lugar de una rotación de cultivos (Montagnini et al., 1992).

La agricultura migratoria constituye el sistema de producción más extendido en las regiones tropicales. Existen muchas variedades de estos sistemas, desde los métodos practicados por tribus nómadas hasta las prácticas más complejas de poblaciones más estables. El barbecho mejorado se considera como una fase intermedia entre la agricultura migratoria y la agricultura sedentaria, con rotaciones más cortas (Raintree, 1987). Raintree y Warner, (1986) hacen distinción entre el barbecho económicamente mejorado, donde los árboles son introducidos por su valor económico, y el barbecho biológicamente mejorado, donde las plantas son introducidas por su capacidad de mejorar la fertilidad del suelo o deprimir el crecimiento de malezas. 


\section{¿Por qué se hace necesario el período de barbecho?}

Después de la tumba y quema, los cultivos que se introducen mantienen una productividad elevada, ya que disminuye la acidez del suelo y aumenta su fertilidad. Después de 2-3 años de cultivo, al aumentar las poblaciones de plagas y malezas, crece la demanda por el uso de nutrimentos, se empobrecen los suelos, aumentan los costos de desmalezado y disminuye la productividad de los cultivos. A partir de estas condiciones los agricultores abandonan las parcelas y comienza el período de barbecho, donde se restablece el reciclaje de nutrimentos, al ser invadidas las parcelas por la vegetación secundaria. Al transcurrir el tiempo las propiedades del suelo vuelven a ser adecuadas para el cultivo.

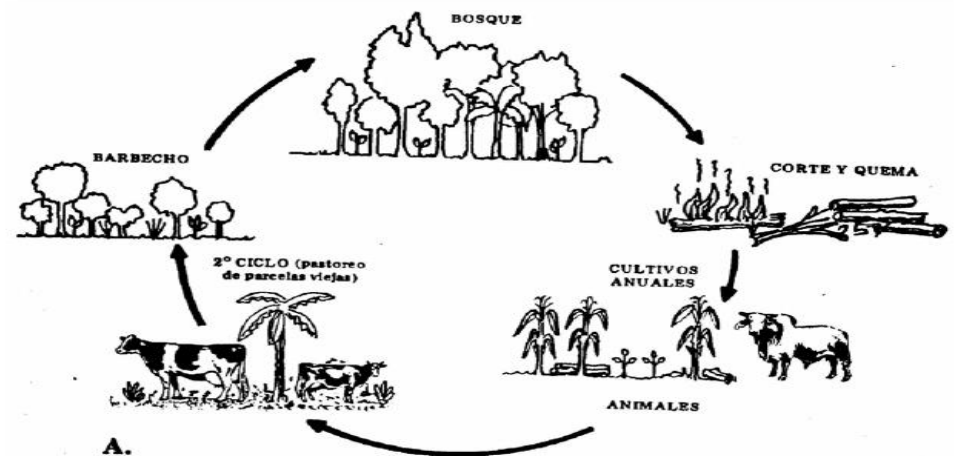

Figura 3. Esquema del ciclo de una parcela manejada con enfoque migratorio

Este tipo de agricultura puede ser una forma ecológica y económicamente racional del uso de recursos, cuando la densidad y la presión de la población sobre el uso de la tierra son bajas y los períodos de barbecho son lo suficientemente largos como para restablecer la fertilidad del suelo. Habitualmente se practica en condiciones en que la mano de obra es más escasa que la tierra, el capital disponible escaso y el nivel de tecnología bajo. En Cuba se practican dos formas de barbecho, una asociada a la recuperación de los suelos erosionados, principalmente en las zonas alomadas y montañosas, y la otra relacionada con la alta infestación por aroma (Cassia farnesiana) y marabú (Dichrostachys cinerea) que presentan el $56 \%$ de los suelos agropecuarios del país (Paretas et al., 1996).

En el primer caso el sistema consiste en proporcionar 1 ó 2 años de descanso a las parcelas de cultivo, dejando que en ellas crezca la vegetación natural y se 
forme paulatinamente un monte secundario o terciario. Una rotación adecuada de las parcelas permite segregar cada año una nueva; con este método se recupera en parte la fertilidad perdida por el cultivo continuo y se incrementa el contenido de materia orgánica del suelo. El segundo sistema se practica de forma generalizada en la actualidad por campesinos llamados "parceleros" o "conuqueros", los cuales desmontan pequeñas áreas infestadas de marabú y aroma para la siembra de cultivos anuales, principalmente arroz (Oryza sativa), maíz, yuca, frijoles (Phaseolus sp.), calabaza, etc.

Después del desmonte los campesinos usan la leña obtenida para la producción de carbón y comienzan un ciclo de cultivos que puede durar de 3-4 años, en dependencia de la pérdida de fertilidad de los suelos, los cuales son muy ricos en nutrientes al inicio de su explotación. Con la disminución de los rendimientos agrícolas los "conuqueros" se desplazan a otras áreas infestadas de marabú, las desbrozan y comienzan su explotación temporal. Este tipo de barbecho practicado en Cuba se diferencia del usado comúnmente en otras zonas de América del Sur y Central en que se involucran en la tala pequeñas áreas de monte, las cuales normalmente están invadidas por especies invasoras y perjudiciales para el desarrollo de la ganadería y/o la agricultura, por lo que el daño desde el punto de vista forestal es mínimo. La otra diferencia consiste en que el barbecho no es planificado ni está controlado en el tiempo, por lo que existe la posibilidad de utilizar nuevamente las áreas en reposo en un período corto, lo que implica que el descanso no sea lo suficientemente largo como para que se recupere el nivel de fertilidad adecuado y eventualmente se pueden producir problemas de degradación de los suelos.

\section{ÁRBOLES EN ASOCIACIÓN CON CULTIVOS ANUALES}

Estos sistemas presentan las mismas interacciones entre los cultivos y los árboles que las asociaciones de árboles con cultivos perennes; sin embargo, para el caso particular de los sistemas de cultivos de callejones también se pueden utilizar especies que no son tolerantes a la sombra. En estos se incluyen cultivos tales como maíz, frijol, guisantes, soya, maní, tubérculos y raíces en asociación con 
árboles, que en la mayoría de los casos son fijadores de nitrógeno. Según Jiménez et al., (1998) los cultivos en callejones son prácticas agroforestales en las que los cultivos anuales son sembrados en los espacios que quedan entre las líneas de una especie leñosa, generalmente leguminosa, que es podada a intervalos regulares para evitar la competencia y proveer un "mantillo" o "mulch", el cual sirve para controlar las malezas y a su vez proveer nutrimentos al suelo. Alternativamente, el material podado puede ser utilizado como leña o alimento para el ganado. Los beneficios potenciales de este sistema son los siguientes (Kang y Wilson, 1987): 1. Las prácticas de cultivo y barbecho se realizan simultáneamente. 2. Se aumenta el período de cultivo y la intensidad del uso de la tierra. 3. Se logra una regeneración efectiva de la fertilidad del suelo con especies más eficientes para este propósito. 4. Requerimientos bajos de insumos externos. 5. El sistema es de escala neutral y lo suficientemente flexible para su uso por agricultores pequeños y para la producción mecanizada a gran escala.

Para el establecimiento de cultivos en callejones generalmente se utilizan leguminosas arbóreas, pues muchas de estas presentan un rápido crecimiento, alta producción de biomasa de fácil descomposición, respuesta a las podas, alta capacidad de rebrote y capacidad de fijar el nitrógeno atmosférico. Su establecimiento se puede realizar por semillas o de forma asexual, aunque generalmente se utiliza el segundo método, con estacas de aproximadamente 1,5 $\mathrm{m}$ de largo y un diámetro mínimo de 4-8 cm. Una vez establecidos los árboles, la decisión de establecer un programa de podas es lo más importante. El momento de la poda lo determina el cultivo agrícola, ya que del árbol se obtiene el aporte de nutrimentos, pero a la vez se debe minimizar la competencia por la luz, el agua y los nutrimentos que este pudiera ejercer sobre el cultivo. Según Jiménez et al., (1998) se debe podar en mayo para permitir la siembra y el desarrollo de los cultivos durante 90-120 días de las lluvias y luego practicar una poda en septiembre $u$ octubre, la cual puede continuarse con barbechos o siembra de nuevos cultivos según las condiciones agroecológicas del lugar Tabla 2). 
Tabla 2. Arreglo cronológico según los cultivos y la época

\begin{tabular}{|c|c|c|c|c|c|c|c|c|c|c|c|c|}
\hline \multirow{2}{*}{$\begin{array}{l}\text { Alternativas } \\
\text { por meses }\end{array}$} & \multicolumn{12}{|c|}{ Meses } \\
\hline & 5 & 6 & 7 & 8 & 9 & 10 & 11 & 12 & 1 & 2 & 3 & 4 \\
\hline$A$ & $P$ & $\mathrm{C}_{1}$ & $\mathrm{C}_{1}$ & $\mathrm{C}_{1}$ & $P$ & $\mathrm{C}_{2}$ & $\mathrm{C}_{2}$ & $\mathrm{C}_{2}$ & $\mathrm{P}^{*}$ & B & B & $B$ \\
\hline $\mathrm{B}^{*}$ & $P$ & $\mathrm{C}_{1}$ & $\mathrm{C}_{1}$ & $\mathrm{C}_{1}$ & $C_{1}$ & B & $B$ & $P$ & $\mathrm{C}_{2}$ & $\mathrm{C}_{2}$ & $\mathrm{C}_{2}$ & B \\
\hline$C$ & $P$ & $C_{1}$ & $\mathrm{C}_{1}$ & $\mathrm{C}_{1}$ & $C_{1}$ & $\mathrm{P}^{*}$ & B & B & $B$ & $B$ & $B$ & B \\
\hline
\end{tabular}

P-poda. $\mathrm{P}^{*}$-poda opcional. C1- primer cultivo. A-Trópico seco C2-cultivo de segunda. B*Trópico húmedo. B-barbecho. C-Extrema sequía

Fuente: Adaptado de Jiménez et al., (1998).

En nuestra opinión y teniendo en cuenta las condiciones de precipitación de Cuba, es factible utilizar la variante $A$ en la zona occidental, ya que todavía en los meses de septiembre y octubre ocurren precipitaciones asociadas a los frentes fríos que permiten un desarrollo adecuado del cultivo de segunda; sin embargo, en la zona oriental sería conveniente emplear la variante $\mathrm{C}$, donde el barbecho y la precipitación del período seco beneficiarían grandemente la fertilidad de los suelos. El sistema de cultivo en callejones no se ha difundido en el país, ya que anteriormente la producción de cultivos temporales estaba asociada a la utilización de altos insumos; sin embargo, en las condiciones actuales de crisis económica y pérdida de mercados externos dicho sistema puede ser una alternativa viable para suplir el déficit de fertilizantes, principalmente nitrogenados, con la variante de incorporar al suelo el material podado, en lugar de dejarlo sobre la superficie.

La experiencia de otros países recomienda el uso de las siguientes especies para el desarrollo de cultivos en callejones: G. sepium, L. leucocephala, Cajanus spp., E. poeppigiana, Inga edulis, Cassia spp., Prosopis spp. y Calliandra spp. Se plantea que el éxito del uso de este sistema agroforestal está asociado a su adaptación a las necesidades de los agricultores, tanto en el manejo del terreno como en los productos que se puedan obtener para el autoconsumo o las necesidades alimenticias de una localidad (Jiménez et al., 1998), por lo que es necesario sembrar cultivos y árboles de un alto valor comercial y que a su vez se complementen ecológicamente. 


\section{CONCLUSIONES}

1. La definición del término Agroforestería es bastante reciente, y aunque numerosos autores lo enfocan desde diferentes ángulos y posiciones, consideramos que el concepto del International Council for Research in Agroforestry es lo suficientemente claro y conciso para ser tomado como referencia en un debate internacional. No obstante, aunque la frase "rendimiento sostenido" se usa frecuentemente como una cualidad obligada de la agroforestería, no existe un planteamiento claro, en el concepto como tal, acerca del cuidado de la naturaleza, de la conservación del entorno a largo y mediano plazo, aspecto muy importante en los momentos actuales cuando el mundo se debate ante la perspectiva de la desaparición de las fuentes de energía no renovables, así como de numerosas especies de animales y plantas y del tesoro más preciado: el hombre. Aunque en la literatura revisada está explícita la importancia del enfoque medioambientalista en las investigaciones agroforestales y los sistemas de uso de la tierra, sería interesante llegar a un concepto de Agroforestería en el cual un elemento condicionante sea la protección de los recursos naturales, sin la cual no es posible un rendimiento sostenido de ningún sistema de producción.

2. La clasificación de los sistemas agroforestales es muy diversa y está relacionada con los componentes que los integran, la estructura en el espacio, el diseño a través del tiempo, los objetivos de producción y las características bioeconómicas y sociales prevalecientes.

3. Los sistemas agroforestales que combinan el uso de cultivos con especies leñosas sin la intervención directa de la ganadería están muy difundidos en el mundo; los más representativos y estudiados son: los sistemas Taungya, los huertos caseros mixtos tradicionales, los árboles en asociación con cultivos perennes y anuales (cultivo en callejones) y la agricultura migratoria con intervención o manejo de barbechos. 
4. En las condiciones de Cuba los sistemas agroforestales están bien representados en diferentes formas de uso de la tierra e incluso existen sistemas muy propios, los cuales han surgido debido a situaciones específicas del contexto agropecuario cubano. Tal es el caso de la producción de cultivos anuales en áreas de fomento de sistemas silvopastoriles para la ganadería vacuna y la agricultura migratoria practicada por campesinos "parceleros" individuales.

5. En los últimos años los sistemas agroforestales han tenido el éxito de ser ampliamente aceptados en muchos países del trópico. La razón principal de esta aceptación ha sido su alta adaptabilidad a diferentes condiciones ambientales y socioeconómicas, una flexibilidad de estrategias y prácticas y su orientación directa hacia la solución de los problemas. En el caso de Cuba los sistemas agroforestales constituyen alternativas viables para nuestro escenario rural, con vistas a satisfacer de forma sostenida las necesidades elementales de alimentación y las condiciones de vida de la población, mediante diferentes formas de adopción según las características propias del ecosistema donde se implanten.

6. Las prioridades de investigación en el futuro deben estar encaminadas a abordar, con un enfoque sistémico, las relaciones ecológicas, económicas y sociales de los sistemas agroforestales, con el objetivo de encontrar métodos alternativos de uso de la tierra que permitan solucionar los problemas de la deforestación, la falta de madera de uso directo y de combustible, la escasez de alimentos agrícolas para la población y su comercialización, la baja fertilidad de los suelos y su alta tasa de erosión, así como otros aspectos sociales entre los que se encuentran el éxodo del campo a la ciudad y la pérdida del sentido de pertenencia hacia la tierra.

\section{REFERENCIAS BILIOGRÁFICAS}

1. Aguire, C.A. 1963. Estudio silvicultural y económico de sistema Taungya en condiciones de Turrialba. Tesis M. Sc. CATIE. Turrialba, Costa Rica. 101 p.

2. Altieri, M.A. 1991. Traditional farming in Latin America. The Ecologist. 21:93

3. ANON. 1981. What is agroforestry?. (Editorial). Agroforestry Systems. 1:7

4. Beer, J. 1998. Ventajas, desventajas y características deseables en los árboles de sombra para café, cacao y té. En: Apuntes de clase del curso corto Sistemas Agroforestales. (Eds. F. Jiménez y A. Vargas). Proyecto Agroforestal CATIE/GTZ. Turrialba, Costa Rica. p. 169 
5. Beer, J.; Kapp, G.; Lucas, C. 1994. Alternativas de reforestación: Taungya y sistemas agrosilviculturales permanentes vs plantaciones puras. CATIE. Turrialba, Costa Rica. Serie Técnica. Informe Técnico No. 230. 25 p.

6. Bene, J.G.; Beal, H.W., Cote, A. 1977. Trees, food and people: land management in the tropics. I.D.R.C. Ottawa, Canadá. 59 p.

7. Budowski, G. 1984. Los sistemas agroforestales en América Central. En: Agroforestería. Actas del Seminario. (Eds. J. Heuveldop y J. Lagemann). CATIE. Turrialba, Costa Rica. 112 p.

8. Budowski, G. 1990. Agroforestería en Costa Rica y su relación con el manejo de suelos. Conferencia. Universidad de Costa Rica. San José, Costa Rica. 5 p.

9. Camacho, Y. 1992. Mediciones del componente arbóreo y cercas vivas y cortinas rompevientos. Conferencia. Curso Internacional IICA-CATIE de especialización en desarrollo de sistemas agroforestales. CATIE, Turrialba, Costa Rica. $17 \mathrm{p}$

10. Christant Y. L. 1990. Home gardens in tropical Asia, with special reference to Indonesia. In: Tropical home gardens. (Eds. K. Landauer and M. Brazil). United Nations University Press. Tokyo, Japan. p. 9

11. Combe, J.; BudowskI, G. 1979. Classification of agroforestry techniques. (Ed. G. de las Salas). Proc. Symp. Agroforestry Systems in Latin America. CATIE. Turrialba, Costa Rica, p. 17

12. FAO. 1984. Sistemas agroforestales en América Latina y el Caribe. Oficina Regional para América Latina y el Caribe. Santiago de Chile, Chile. s. p.

13. Fassbender, H.W. 1993. Modelos edafológicos de sistemas agroforestales. Segunda edición. Proyecto agroforestal CATIE/GTZ. Turrialba, Costa Rica. Serie de Materiales de Enseñanza No. 29. 491 p.

14. Geilfus, F. 1989. El árbol al servicio del agricultor. Manual de Agroforestería para el desarrollo rural. Vol. 2. Guía de especies. ENDARA-CARIBE. CATIE. Santo Domingo, República Dominicana. $86 \mathrm{p}$

15. Hernandez, I.; Simon, L. 1993. Los sistemas silvopastoriles: empleo de la agroforestería en las explotaciones ganaderas. Pastos y Forrajes. 16:99

16. INTERNATIONAL COUNCIL FOR RESEARCH IN AGROFORESTRY. 1983. Guidelines for agroforestry diagnosis and design. Nairobi, Kenya. $25 \mathrm{p}$.

17. Jimenez, F.; Vargas, A. 1998. Apuntes de clases del curso corto Sistemas Agroforestales. Proyecto Agroforestal CATIE/GTZ. Turrialba, Costa Rica. $359 \mathrm{p}$.

18. Jlmenez, J.; Kass, D.; Jimenez, F. 1998. El cultivo en callejones. En: Apuntes de clases del curso corto Sistemas Agroforestales. (Eds. F. Jiménez y A. Vargas). Proyecto agroforestal CATIE/GTZ. Turrialba, Costa Rica. p. 257

19. Kang, B.T.; Wilson, G.F. 1987. The development of alley cropping as a promising agroforestry technology. In: Agroforestry: a decade of development. (Eds. H.A. Steppler and P.K.R. Nair). International Council for Research in Agroforestry. Nairobi, Kenya. p. 227

20. Kass, D.C.L. 1992. Agroforestales. Conferencia Curso Internacional "Desarrollo de Sistemas Agroforestales". CATIE. Turrialba, Costa Rica. 5 p. (Mimeo)

21. Lamprecht, H. 1990. Silvicultura en los trópicos: los ecosistemas forestales en los bosques tropicales y sus especies arbóreas; posibilidades y métodos para un aprovechamiento sostenido. Deutsche Gesellschaft für Technische Zusammenarbeit. Rossdorf, Alemania. 335 p.

22. Lojan, L. 1979. Sistemas agroforestales en el sur del Ecuador. (Ed. G. de las Salas). Actas. Taller Sistemas Agroforestales en América Latina. CATIE. Turrialba, Costa Rica. p. 132

23. Lok, Rossana. 1998. Introducción a los huertos caseros tradicionales tropicales. Proyecto Agroforestal CATIE/ GTZ. Turrialba, Costa Rica. Módulo de Enseñanza Agroforestal No. 3. $157 \mathrm{p}$.

24. Lucas, C.; Beer, J.; Kapp, G. 1995. Reforestación con maderables. Sistemas agrosilviculturales vs plantaciones puras en Talamanca, Costa Rica. Resultados agrícolas y forestales. CATIE. Turrialba, Costa Rica. Serie Técnica. Informe Técnico No. 243. 65 p.

25. Martinez, H.A. 1989. El componente forestal en los sistemas de finca de pequeños agricultores. CATIE. Turrialba, Costa Rica. $79 \mathrm{p}$.

26. Maydell, H.J. Von. 1984. Los sistemas agroforestales desde el punto de vista forestal. En: Agroforestería. Actas del Seminario. (Eds. J. Heuveldop y J. Lagemann) CATIE. Turrialba, Costa Rica. 112 p. 
27. Montagnini, F; prevetti, L; Thrupp, L A; beer, J.; BoreL, R.; Budowskl, G.; Espinoza, L.; heuveldop, J.; Reiche, C.; Russo, R.; Salazar, R.; Alfaro, M; Rojas, I; Berstch, F; Fernandes, E.; Gonzalez, M.; Alvim, R.; Shaheduzzaman, MD.; NIchols, D. 1992. Sistemas agroforestales. Principios y aplicaciones en los trópicos. Organización para Estudios Tropicales (OET). San José, Costa Rica. 622 p.

28. Nair, P.K.R. 1984. Soil productivity aspects of agroforestry. ICRAF. Science and Practice of Agroforestry No. 1.85 p.

29. Nair, P.K.R. 1985. Classification of agroforestry systems. Agroforestry Systems. 3:97

30. Nair, P.K.R. 1989. Agroforestry defined. In: Agroforestry systems in the tropics. (Ed. P.K.R. Nair). Kluwer Academic Publishers. Dordrecht, The Netherlands. p. 13

31. Nair, P.K.R. 1993. An introduction to agroforestry. Kluwer Academic Publi-shers. Dordrecht, The Netherlands. p. 85

32. OFFICE OF TECHNOLOGY ASSESSMENT. 1984. Technologies to sustain tropical forest resources. U.S. Congress. Washington D.C. p. 9-19, 219-223

33. Orquin, J.; Rlvero, M.P.; Gainza, A. 1988. Estudio preliminar de asociaciones compuestas por Musa sapientum, Coffea arabica, Dioscorea alata y Pinus cubensis en la zona de Baracoa. Taller Agroforestal No. 1. Estación Experimental Forestal de Guisa. Granma, Cuba. 16 p.

34. Paretas, J.; Mesa, B.; Lopez, M.; Rodriguez, V.; Abreu, D.; Gonzalez, A. 1996. Marabú-aroma II. Obstáculos al rescate ganadero. Programa de control y sus resultados. ACPA. 1:48

35. Pezo, D.A. 1991. La producción ganadera en un contexto agroforestal. Revista El Chasqui. 25:1

36. Platen, H. V. 1996. Alternativas de reforestación. Taungya y sistemas agrosilviculturales permanentes vs plantaciones puras; la economía. CATIE. Turrialba, Costa Rica. Serie técnica. Informe técnico No. 250. 55 p.

37. Raintree, J.B. 1987. Factores que afectan la adopción de innovaciones agroforestales por agricultores tradicionales. En: Avances en investigación agroforestal. Memoria del Seminario. (Eds. J. Beer, H. W. Fassbender y J. Heuveldop). CATIE/ GTZ. Turrialba, Costa Rica. p. 307

38. Raintree, J.B.; Warner, K. 1986. Agroforestry pathways for intensification of shifting agriculture. Agroforestry Systems. 4:39

39. Renda, A.; calzadilla, E.; Jimenez, M; Sanchez, J. 1997. La Agroforestería en Cuba. Red Latinoamericana de Cooperación Técnica en Sistemas Agroforestales. Dirección de Recursos Forestales. FAO, Roma-Oficina Regional de la FAO para América Latina y el Caribe. Santiago de Chile, Chile. 64 p.

40. Schlönvoigt, A. 1998. Sistemas Taungya. Módulo de Enseñanza Agroforestal No. 4. CATIE. Turrialba, Costa Rica. 116 p.

41. Simon, L. 1998. Experiencia práctica del silvopastoreo en condiciones de la producción. Conferencia del Diplomado en Silvopastoreo. EEPF "Indio Hatuey". Matanzas, Cuba. 9 p. (Mimeo)

42. Somarriba, E. 1998. ¿Qué es agroforestería? En: Apuntes de clase del curso corto Sistemas Agroforestales. (Eds. F. Jiménez y A. Vargas). Proyecto Agroforestal CATIE/GTZ. Turrialba, Costa Rica. p. 3

43. Wiersum, K.F. 1981. Outline of the agroforestry concept. In: Viewpoints in agroforestry. (Ed. K.F. Wiersum). Agricultural University of Wageningen, The Netherlands. p. 1

44. Young, A. 1989. Agroforestry for soil conservation. CAB International/ ICRAF. Science and Practice of Agroforestry No. 4. 276. 